\title{
Fluctuations of the electromagnetic local density of states as a probe for structural phase switching
}

\author{
N. de Sousa, ${ }^{1,2}$ J. J. Sáenz, ${ }^{2,3}$ F. Scheffold, ${ }^{4}$ A. García-Martín, ${ }^{5}$ and L. S. Froufe-Pérez ${ }^{4, *}$ \\ ${ }^{1}$ Departamento de Física de la Materia Condensada, Universidad Autónoma de Madrid, 28049 Madrid, Spain \\ ${ }^{2}$ Donostia International Physics Center (DIPC), Paseo Manuel Lardizabal 4, 20018 Donostia-San Sebastian, Spain \\ ${ }^{3}$ IKERBASQUE, Basque Foundation for Science, 48013 Bilbao, Spain \\ ${ }^{4}$ Physics Department, University of Fribourg, Chemin du Musée 3, CH-1700 Fribourg, Switzerland \\ ${ }^{5}$ IMM - Instituto de Microelectrónica de Madrid (CNM-CSIC), Isaac Newton 8, PTM, Tres Cantos, E-28760 Madrid, Spain
}

(Received 13 June 2016; published 19 October 2016)

\begin{abstract}
We study the statistics of the fluorescence decay rates for single quantum emitters embedded in a scattering medium undergoing a phase transition. Under certain circumstances, the structural properties of the scattering medium explore a regime in which the system dynamically switches between two different phases. While in that regime the light-scattering properties of both phases are hardly distinguishable, we demonstrate that the lifetime statistics of single emitters with low diffusivity is clearly dependent on the dynamical state in which the medium evolves. Hence, lifetime statistics provides clear signatures of phase switching in systems where light scattering does not.
\end{abstract}

DOI: 10.1103/PhysRevA.94.043832

\section{INTRODUCTION}

The sensitivity of the spontaneous-emission rate of an excited dipole emitter to the local environment [1] makes singlemolecule spectroscopy a unique tool to sense optical and structural properties in its surroundings on the nanoscale [2-6]. Control of the emission rate has been demonstrated using a variety of well-defined structures, such as metal surfaces [7], cavities [8], photonic crystals [9], and nanoantennas [10]. Understanding the basic physics of spontaneous-emission rates in complex media is of paramount importance for many applications (molecular imaging techniques [2-6], solar cells [11], laser technology [12,13], and single-photon sources [14]), which explains the increasing interest in their statistical properties in random environments [15-21].

From a fundamental point of view, the emission rate is proportional to the number of optical modes available for emission at the position of the emitter, i.e., proportional to the electromagnetic local density of states (LDOS) [22]. In a complex disordered medium, the LDOS presents strong fluctuations due to dynamic conformational fluctuations of the system around the emitter or when the emitter itself diffuses through it [3-5]. The statistical fluctuations of the LDOS $[15,17,23]$ are directly linked to the so-called $C_{0}$ speckle correlations [24,25]. In the absence of spatial correlations, the averaged LDOS and the transport extinction mean free path $\ell$ are linked through causality Kramers-Kronig relations [26], and the LDOS fluctuations $C_{0}$ were predicted to increase with the scattering strength $\sim \ell^{-1}$ [24]. However, the correlations between the emitter position and the surrounding scatterers, due to the unavoidable excluded volume around the emitter, make the LDOS and its fluctuations strongly nonuniversal [25] and sensitive to both $\ell$ and the local correlation length $[16,17,25,27]$.

The near-field effects on the LDOS close to a single particle are relatively well understood [28]. In random media, when the positions of the scatterers around the emitter are

\footnotetext{
*luis.froufe@unifr.ch
}

not correlated, numerical simulations show that the LDOS fluctuations can be explained to a large extent by a single scattering statistical model [16] and are dominated by the near-field interaction with the nearest scatterer at the scale of the excluded volume [16,17]. Temporal lifetime fluctuations can then be correlated to fluctuations in the position of the nearest scatterer and provide a suitable probe for the dynamics of the structure around the emitter [5]. In particular, the predicted non-Gaussian long-tailed distributions of emission rates in disordered dielectrics [16,17] are compatible with experimentally measured ones [21].

However, similar experiments do not show such long-tailed distributions [20]. This result has been attributed to finitesize effects in the scatterers. Recent experiments [29] also suggest that hydrophobic interaction between the scatterers and the solvent in a colloidal suspension plays an important role in the description of the decay-rate and quantum yield statistics. On the other hand, structural correlations in the disorder structure have a profound effect on the lifetime statistical distributions [17,30]. All of the reported results show that the near-field scattering plays an essential role in the description of lifetime statistics in disordered media. Nearfield effects also have important consequences in mesoscopic light transport [31,32].

In this work, we show that the statistics of emission rates in correlated disordered media is extremely sensitive to the details of the radial distribution function around the emitter. We analyze the emission statistics for a single emitter embedded in a finite cluster of resonant particles in a model system similar to that described in previous works [17]. However, instead of generating random configurations of scatterers [16,17], we compute the emission rates as the system evolves with time under equilibrium conditions. Assuming a standard Lennard-Jones (L-J) interaction between particles, this system is known to present a peculiar solid-liquid-like phase transition at finite temperature: Due to finite-size effects, the two phases cannot coexist at the melting temperature and the whole cluster presents an interesting dynamical behavior, switching between an amorphous solidlike phase and liquidlike phases [33,34]. This makes it an ideal model system to analyze the effects of 


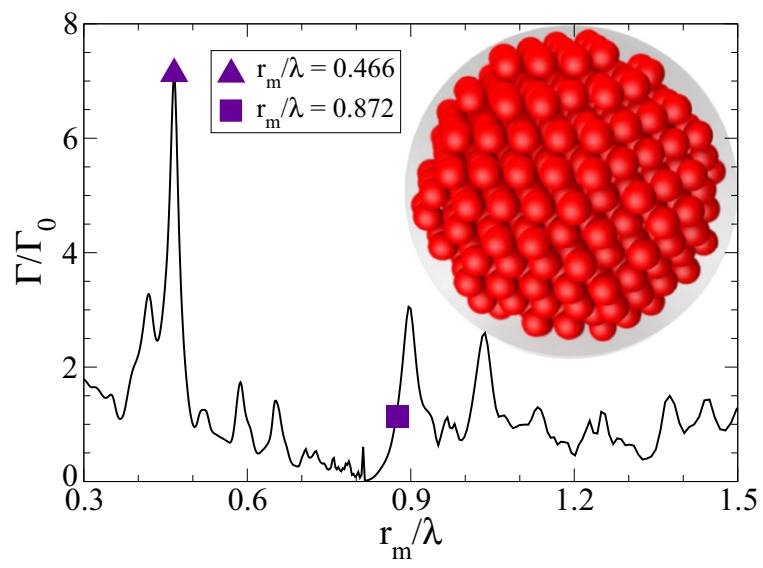

FIG. 1. Normalized decay-rate (equal to the LDOS normalized to the vacuum one) spectrum of a single emitter placed at the center of a cluster at $T=0$ (see text for further details). Inset: the system under study. Each point scatterer is replaced by a sphere of radius $r_{m} / 2$. The translucid sphere represents the confining sphere.

local order on the emission rates. At very low temperatures, the system is a well-ordered structure that in the limit of infinite size, would correspond to a face-centered-cubic lattice. Due to this order, the spectrum of emission rates presents a strong chromatic dispersion reminiscent of the band structure of an infinite crystal of resonant dipoles [35], including spectral windows where the emission is enhanced and pseudogaps where it is dramatically inhibited [36,37]. At the melting temperature, the total scattering cross section of the system does not present significant differences between the two phases, while the emission rate jumps following the dynamic of the system. While light-scattering measurements would be blind to such dynamical changes, the lifetime statistics would then provide a direct signature of a phase-switching behavior.

\section{STRUCTURAL MODEL AND LIGHT SCATTERING STATISTICS}

In our model system, sketched in the inset of Fig. 1, we consider a three-dimensional cluster of $N=515$ particles confined inside a spherical cavity. The particles interact through a Lennard-Jones (L-J) potential:

$$
V_{L J}(r)=\varepsilon\left[\left(\frac{r_{m}}{r}\right)^{12}-2\left(\frac{r_{m}}{r}\right)^{6}\right],
$$

where $\varepsilon$ is the depth of the potential well, $r$ is the distance between particles, and $r_{m}$ is the equilibrium distance of the potential. The confining spherical volume is chosen in such a way that near crystal density is achieved [34].

From the ensemble of $N$ particles, the one closest to the center of the distribution is considered to be a point emitter. The remaining $N-1$ particles are considered to be resonant light scatterers with an electric polarizability, $\alpha=i 6 \pi / k^{3}$ (where $k=2 \pi / \lambda$ is the light wave number and $\lambda$ the wavelength). The electrodynamic response is obtained by using a coupled dipole method described elsewhere [16,17] (which involves the solution of $3 N$ self-consistent multiple scattering equations; see Appendix A). We compute both the total scattering cross section (assuming an external incoming plane wave) and the
LDOS at the emitter position; details of both computations are given in Appendix A. The vacuum normalized LDOS is also the ratio $\Gamma / \Gamma_{0}$ of the emission decay rate $\Gamma$ of a point emitter (placed at the considered position and emitting at the considered wavelength $\lambda$ ) to its emission decay rate in vacuum $\Gamma_{0}$. In Fig. 1, we plot the normalized LDOS at the center of the cluster (after complete relaxation of the structure at $T=0$ ), as a function of $r_{m} / \lambda$, i.e., the ratio between the potential equilibrium distance $r_{m}$ and the emission wavelength $\lambda$.

The rich, peaked structure in this pseudospectrum (reminiscent of the band structure of an infinite crystal of resonant dipoles [35]) is a consequence of the interplay between diffraction and multiple scattering effects of light with the crystal structure, enhanced by the resonant character of the scatterers. We highlight two representative points in the decay-rate pseudospectrum: $r_{m} / \lambda=0.466$, where the decay rate is much larger than in vacuum, and $r_{m} / \lambda=0.872$, where the decay rate is similar to the one in vacuum.

In order to generate a suitable statistical ensemble at fixed temperature, we perform standard dynamic Monte Carlo (DMC) simulations [38] using the canonical ensemble. We depart from a crystalline structure and perform $10^{8}$ of DMC steps (single-particle moves) to thermalize the system. After this process, an extensive DMC sampling is performed computing the scattering efficiency and LDOS for $2 \times 10^{4}$ configurations; each of these configurations is obtained after $10^{5}$ single-particle DMC steps. Details of the statistical DMC simulations are given in [34]. If the temperature of the system is $\tilde{T}$, we define a normalized temperature $T \equiv K_{B} \tilde{T} / \varepsilon$, where $K_{B}$ is Boltzmann's constant, and $\varepsilon$ is the L-J potential-well depth. In particular, at temperature $T=0.6 \equiv T_{m}$, the system presents the aforementioned dynamical phase switching between low-energy (solidlike) and high-energy (liquidlike) branches. In Fig. 2(a), we plot the energy per particle sampling as a function of the number of DMC cycles and a switch event from high to low energy is clearly observed. The average of the self-diffusion coefficients was found to largely vary from the liquidlike to the solidlike phases, providing an unambiguous signature of the actual phase state [34]. Interestingly, the same simulations showed that the pair-correlation function $g(r)$ is essentially the same for both phases, as shown in Fig. 2(b) [34]. This indicates that the system switches from liquid to an amorphous solid phase rather than crystal-like and suggests that light-scattering experiments could not be sensitive to this subtle dynamical switching. As a matter of fact, this is consistent with our numerical results shown in Fig. 2(c), where we present the energy sampling versus the computed normalized scattering cross section $Q_{\text {scat }}$ (scattering efficiency) for $r_{m} / \lambda=0.872$. To guide the eye, points corresponding to high and low internal energy are rendered in different colors. Integrating the sampling in energy, we obtain scattering efficiency histograms, as shown in Fig. 2(d). Differences in $Q_{\text {scat }}$ histograms corresponding to high- and low-energy phases can hardly be distinguished. The $Q_{\text {scat }}$ histogram obtained by considering all the values of $Q_{\text {scat }}$ for all possible energies [shaded gray area in Fig. 2(d)] shows a single peak and no signature of the two-state switching.

It is well known that positional correlations between scatterers can strongly affect the wave-transport properties, i.e., the transport mean free path, in bulk disordered media. 
They are responsible, for example, for the large conductivity of liquid metals [39], the cornea transparency [40], the strong chromatic dispersion in colloidal suspensions [41] and amorphous photonic materials [42], and natural structural coloration [43]. The correlations in wave transport through a translationally invariant system are encoded in the paircorrelation function $g(r)$. As expected, we conclude that lightscattering experiments would not provide a way to distinguish between phases in the switching regime due to the indistinguishability of the $g(r)$ in the different dynamical regimes.

\section{LDOS STATISTICS}

Emission decay-rate statistics (or LDOS) shows clear signatures of the phase-switching regime. In Figs. 3(a) and 3(c), we present an energy decay-rate sampling performed at $T=0.6$ at two different ratios of the interaction potential characteristic length to emission wavelength $r_{m} / \lambda$ (the ones highlighted in Fig. 1). In the present model, the point emitter is chosen to be located at the position of the interacting particle closest to the origin. In this way, the dynamics of the emitter and the remaining scatterers is indistinguishable. The direction of the radiating dipole is random and considered to be uniformly distributed among the whole $4 \pi$ angles. We have verified that despite the fact that the spatially averaged self-diffusion constant varies by a factor of three between the two phases, the particle located initially at the center of the cluster hardly diffuses along the DMC calculation. Hence, we can consider the emitter as a low-diffusivity one.
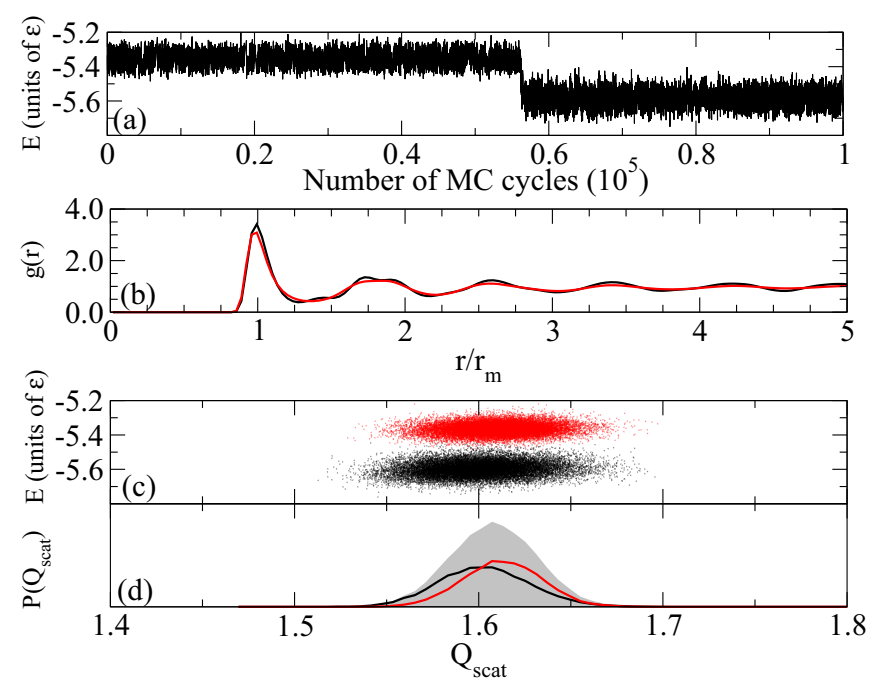

FIG. 2. (a) Energy-per-particle sampling at $T=0.6$ as a function of the MC steps. (b) Corresponding pair-correlation function among scatterers $g(r)$ for the high-energy branch [red (gray)] and for the low-energy branch (black). (c) Energy-scattering efficiency sampling at the switching region $\left(T=0.6, r_{m} / \lambda=0.872\right.$ ); red (gray) dots correspond to high-energy states and black dots correspond to lowenergy states. (d) Corresponding scattering efficiency distributions for the upper energy branch [red (gray) curve] and lower energy branch (black curve) corresponding to liquid and solid phases, respectively. The shaded area corresponds to the sum of both high- and low-energy distributions.

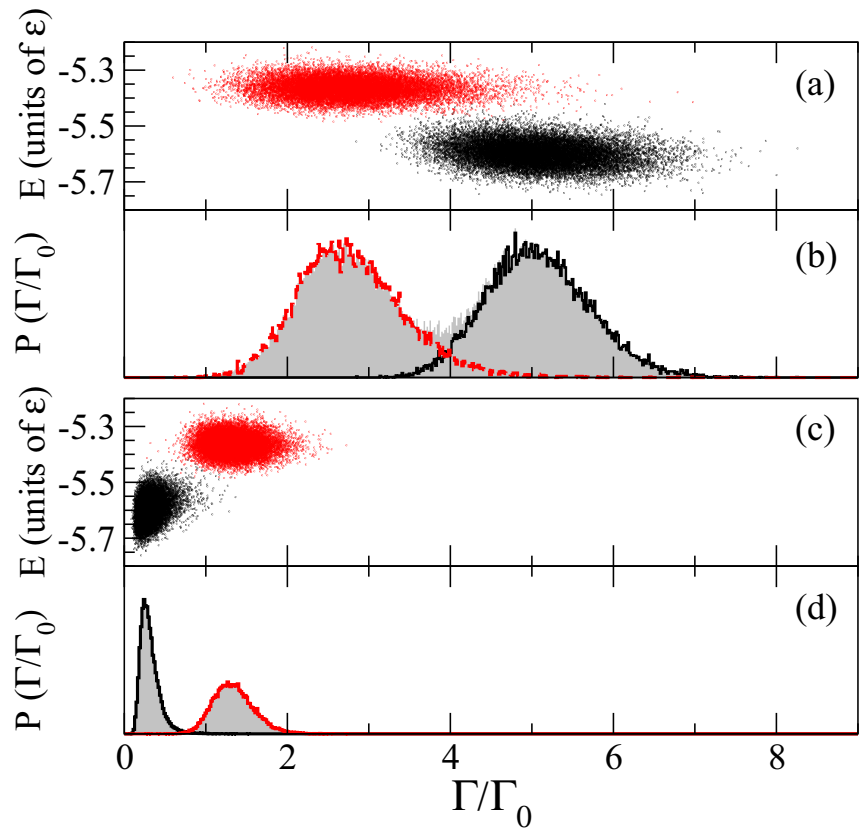

FIG. 3. Energy-per-particle decay-rate sampling at the switching region $(T=0.6)$ for different ratios $r_{m} / \lambda$ : (a) $r_{m} / \lambda=0.466$, (c) $r_{m} / \lambda=0.872$. (b), (d) The corresponding decay-rate distributions are shown after integrating in energies: black histograms correspond to the solid phase, red (gray) ones correspond to the liquid phase, and the shaded gray areas correspond to the total measured decay rates (sum of both solid and liquid distributions).

On the other hand, as discussed in more detail in Appendix A, we calculate the emission decay rates of the dipole emitter considering all the multiple scattering in the system. We nevertheless do not take into account any far-field radiation delay due to radiation trapping. Those effects might be present and would be caused by coupling to long-lived modes into the sample. However, it can be argued that the sample is in the diffusive regime (see Appendix B), where such long-lived modes should be rare. In fact, as demonstrated in [31], at least in collections of uncorrelated disordered point scatterers, such long-lived modes do not exist.

The emission rates evolve with time, following the fast structural changes in the dynamic coexistence region. As it can be observed in Figs. 3(a) and 3(c), the decay-rate distributions corresponding to lower-energetic levels (solid phase, black dots and lines) are different from the higher-energetic levels (liquid phase, red dots and lines). In particular, its average values and fluctuations are appreciably different. Collecting all the emission rates in a histogram results in the statistical distributions of emission rates shown as shaded gray areas in Figs. 3(b) and 3(d). For the selected working wavelengths, the distributions are always bimodal, showing a clear signature of the phase switching.

In order to clarify the origin of the statistical signatures of phase switching in single-emitter decay rates, we analyze in the following the normalized emitter radial distribution function (ERDF) of scatterers around the emitter. The ERDF is defined as the probability of finding a particle at a distance $r$ from the emitter $P(r)$ normalized to the probability in the absence of any correlation $\left(\propto r^{2}\right)$. In this paper, we make a distinction between 


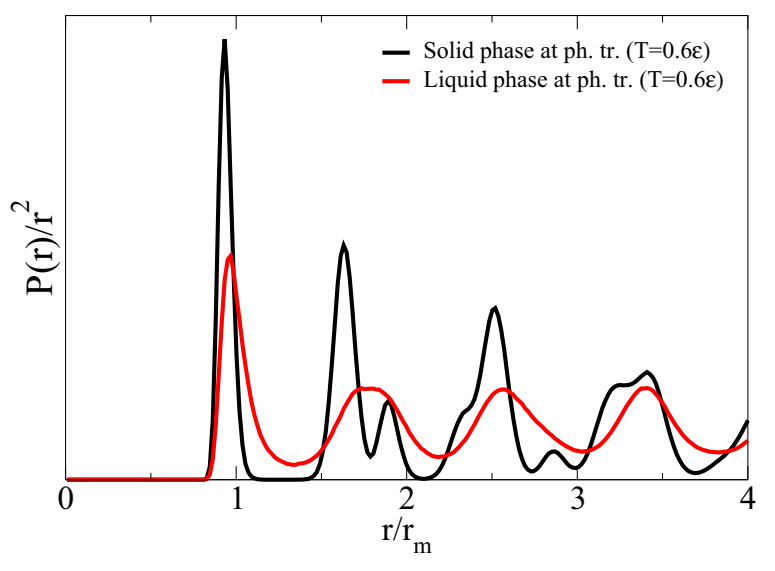

FIG. 4. Emitter radial distribution function (ERDF) for scatterers surrounding the emitter at the phase-switching region $(T=0.6)$. Black and red (gray) curves represent the solid and liquid region, respectively.

ERDF and the pair-correlation function. While for $g(r)$ we consider all pairs of scatterers, we reserve the term ERDF only for the distributions of distances between the emitter and the scatterers. In a translationally invariant system, both distributions should be the same since we consider the emitter to have the same dynamics as the scatterers. Nevertheless, as shown in the next paragraphs, in our relatively small and strongly confined system, the emitter, despite being subjected to the same interaction potential, behaves in a singular way as compared to the remaining scatterers pairs because it is placed very close to the center and, at the temperatures of interest, remains close to its initial position during the course of the simulation.

In contrast to the pair-correlation function $g(r)$, the ERDF at constant temperature shows dramatic variations that follow the phase switching. In Fig. 4, we show the ERDF at $T=0.6$ calculated in the low-energy regime, or amorphous solid phase, and in the high-energy regime, or liquid phase. As can be observed in this figure, the solid phase exhibits a richer peak structure than the liquid phase. This fact might be related to a better layering of the structured around its center. In particular, we observe that the probability of finding particles close to the emitter, represented by the height of the first peak, is much higher in the solid phase than in the liquid one.

With the above considerations, the physical picture we devise is as follows. The ensemble-averaged scattering cross section is determined by the pair-correlation function $g(r)$. Hence, for identical scatterers, similar $g(r)$ shall lead to similar scattering properties. The dynamics of light emission by single emitters, however, is controlled not only by the multiple scattering properties of the whole ensemble, but also by the distribution of scatterers around the emitter, in turn described by the ERDF [16]. Hence, if we have a system showing disparate ERDFs for a slowly diffusing emitter, the lifetime emission statistics of such an emitter can be controlled by the ERDF variations even when the $g(r)$ remains almost unchanged.

\section{CONCLUSIONS}

In summary, we have presented a model system of interacting light scatterers that present a solid-liquid phase transition.
In the case where the system is relatively small (few hundreds of scatterers) and strongly confined, the system presents a phase-switching regime where it switches between phases in its entirety for a certain range of temperatures. We have shown that due to the fact that $g(r)$ functions are nearly indistinguishable between both phases, static light-scattering experiments would not be able to discriminate between phases in the switching regime.

Strikingly, we find that single-emitter decay-rate statistics shows strong signatures of the phase-switching regime. We have correlated this behavior to the difference in the radial distribution functions between scatterers and the emitter position which, in turn, might also be attributed to differences in the self-diffusion of scatterers between both phases. Therefore, this could be experimentally verified in an experiment performed using emitters with low diffusivity.

The system we have considered in this work presents an illustration of one deep difference between light scattering and light emission. Apart from the fundamental implications of this effect, it might be used as a tool for monitoring subtle thermodynamical behaviors in complex systems with sizes comparable to the wavelength of the light source employed in the experiment.

\section{ACKNOWLEDGMENTS}

This research was supported by the Spanish Ministry of Economy and Competitiveness through Grants No. FIS201236113-C03, No. FIS2015-69295-C3-3-P, and No. MAT201458860-P, and by the Comunidad de Madrid (Contract No. S2013/MIT-2740). F.S. and L.S.F.-P. acknowledge funding from the Swiss National Science Foundation through the National Centre of Competence in Research Bio-Inspired Materials.

\section{APPENDIX A: LDOS AND TOTAL SCATTERING CROSS SECTION}

In this Appendix, we present the expressions used to evaluate the electromagnetic local density of states (LDOS) and the total scattering cross sections.

Here we consider a particular frequency $\left(\omega=\omega_{0}\right)$ and an associated particular wave number $\left(k=k_{0}=\omega_{0} / c\right)$ at which dipoles are in resonance with the electromagnetic radiation, meaning that the polarizability is now given by $\alpha=i 6 \pi / k_{0}^{3}$.

The electric field at some position $\mathbf{r}$, generated by the presence of a dipole emitter $\boldsymbol{\mu}$ at some position $\mathbf{r}^{\prime}$, can be obtained by operating the Green tensor over the dipole. This is expressed as

$$
\mathbf{E}(\mathbf{r})=\frac{k^{2}}{\epsilon_{0}} \mathbf{G}_{0}\left(\mathbf{r}, \mathbf{r}^{\prime}\right) \cdot \boldsymbol{\mu},
$$

with $\epsilon_{0}$ being the permittivity of vacuum.

The Green tensor is given by [44]

$$
\begin{aligned}
\mathbf{G}_{0}\left(\mathbf{r}, \mathbf{r}^{\prime}\right)= & \frac{e^{i k R}}{4 \pi R}\left[\left(1+\frac{i k R-1}{k^{2} R^{2}}\right) \mathbb{I}\right. \\
& \left.+\left(\frac{3-3 i k R-k^{2} R^{2}}{k^{2} R^{2}}\right) \hat{\mathbf{R}} \otimes \hat{\mathbf{R}}\right],
\end{aligned}
$$


where $R$ is the modulus of the vector $\mathbf{R}=\mathbf{r}-\mathbf{r}^{\prime}, \hat{\mathbf{R}} \otimes \hat{\mathbf{R}}$ denotes the outer product of $\hat{\mathbf{R}}=\mathbf{R} / R$ by itself, and II is the unit dyadic.

For a system formed by $N$ dipole scatterers, the total electric field at some position $\mathbf{r}$ (outside any scatterer) is given by

$$
\mathbf{E}(\mathbf{r})=\mathbf{E}_{\mathrm{ext}}(\mathbf{r})+\frac{k^{2}}{\epsilon_{0}} \sum_{n=1}^{N} \mathbf{G}_{0}\left(\mathbf{r}, \mathbf{r}_{n}\right) \mathbf{p}_{n}
$$

where $\mathbf{E}_{\text {ext }}(\mathbf{r})$ is the external electric field at the considered position, $\mathbf{r}_{n}$ is the position of the $n$th scatterer, and $\mathbf{p}_{n}$ is the induced dipole located at $\mathbf{r}_{\mathbf{n}}$.

Induced dipoles, $\mathbf{p}_{n}=\epsilon_{0} \alpha \mathbf{E}_{n}$, are obtained by selfconsistently solving the set of $3 \mathrm{~N}$ equations relating the total incoming field exciting the $n$th dipole $\mathbf{E}_{n}$ with the external field and the field radiated from the remaining induced dipoles, which is proportional to the total incoming fields impinging onto each of the remaining induced dipoles:

$$
\mathbf{E}_{n}=\mathbf{E}_{\mathrm{ext}}\left(\mathbf{r}_{n}\right)+k^{2} \alpha \sum_{m \neq n} \mathbf{G}_{0}\left(\mathbf{r}_{n}, \mathbf{r}_{m}\right) \mathbf{E}_{m}
$$

Equation (A4) describes the coupled dipole method [45].

The second term on the right-hand side of Eq. (A3) is the scattered field,

$$
\mathbf{E}_{s}(\mathbf{r})=k^{2} \alpha \sum_{n=1}^{N} \mathbf{G}_{0}\left(\mathbf{r}, \mathbf{r}_{n}\right) \mathbf{E}\left(\mathbf{r}_{\mathbf{n}}\right) .
$$

If the external field is given by Eq. (A1), the total field scattered by the collection of scatterers can then be calculated after solving Eq. (A4) with this external field. The normalized spontaneous decay rate $\Gamma / \Gamma_{0}$ of a dipole emitter $\boldsymbol{\mu}$, in the weak-coupling regime, is given by [44]

$$
\frac{\Gamma}{\Gamma_{0}}=1+\frac{6 \pi \epsilon_{0}}{|\boldsymbol{\mu}|^{2} k^{3}} \operatorname{Im}\left[\boldsymbol{\mu}^{*} \cdot \mathbf{E}_{s}\left(\mathbf{r}^{\prime}\right)\right]
$$

where Im stands for imaginary part, and $\Gamma_{0}$ is the emitter's free-space decay rate.

In order to compute the scattering cross section $\sigma_{\text {scat }}$, we consider an incoming plane wave as the external field, $\mathbf{E}_{\text {ext }}(\mathbf{r})=\mathbf{E}_{0} \exp (\mathbf{k} \cdot \mathbf{r})$. After solving Eq. (A4) with this external field, the induced dipoles are obtained and the total scattering cross section of the system can be written in terms of the induced dipoles $\mathbf{p}_{n}$ as [46]

$$
\sigma_{\text {scat }}=\frac{k^{3}}{\epsilon_{0}^{2}\left|\mathbf{E}_{0}\right|^{2}} \sum_{n, m=1}^{N} \mathbf{p}_{n}^{*} \cdot \operatorname{Im}\left[\mathbf{G}_{0}\left(\mathbf{r}_{n}, \mathbf{r}_{m}\right)\right] \mathbf{p}_{m} .
$$

\section{APPENDIX B: TRANSPORT REGIME}

We used a set of resonant electric point dipoles throughout the manuscript. An important question that might arise is whether the system is in the quasiballistic, diffusive, or localization regimes.

Considering the standard diffusion theory, the transport mean free path $\ell_{\mathrm{tr}}$, in the absence of absorption and anisotropic scattering, can be taken as

$$
\ell_{\mathrm{tr}}^{-1}=\rho \sigma_{\mathrm{scat}}^{(p)},
$$

where $\rho$ is the density of scatterers and $\sigma_{\text {scat }}^{(p)}$ is the singleparticle scattering cross section. The density $\rho$ has been taken to be [34] $\rho=1.07 r_{m}^{-3}$. On the other hand, the scattering cross section at resonance is given by

$$
\sigma_{\text {scat }}^{p}=6 \pi k^{-2} \text {. }
$$

We will now estimate both the optical thickness $b \simeq R / \ell_{\mathrm{tr}}$ and $k \ell_{\text {tr }}$ for the cluster of radius $R$ formed by $N=515$ scatterers. Considering that

$$
R=\left(\frac{3 N}{4 \pi \rho}\right)^{1 / 3}
$$

and combining Eqs. (B1)-(B3), we obtain an optical thickness,

$$
\begin{aligned}
b \simeq R / \ell_{\mathrm{tr}} & =\left(\frac{3 N}{4 \pi}\right)^{1 / 3} \frac{3}{2 \pi} \rho^{2 / 3} \lambda^{2} \simeq 2.48\left(\frac{\lambda}{r_{m}}\right)^{2} \\
& \simeq\left\{\begin{array}{lll}
3.27 & \text { for } & r_{m} / \lambda=0.872 \\
11.44 & \text { for } & r_{m} / \lambda=0.466 .
\end{array}\right.
\end{aligned}
$$

Also, we get

$$
k \ell_{\mathrm{tr}}=\frac{4 \pi^{2}}{3}\left(\frac{r_{m}}{\lambda}\right)^{3} \simeq\left\{\begin{array}{lll}
27.81 & \text { for } & r_{m} / \lambda=0.872 \\
4.20 & \text { for } & r_{m} / \lambda=0.466
\end{array}\right.
$$

We can conclude from the above considerations that the system cannot localize due to the large values of $k \ell_{\mathrm{tr}}$, and that it is well, though not very deep, in the diffusive regime due to its relatively large optical thickness.

Of course, the ratio of the transport time to the natural decay rate of the emitter $\tau_{0}=\Gamma_{0}^{-1}$ will depend on the chosen emitter. In [21], it was argued that in a highly scattering system with $k \ell_{\text {tr }} \simeq 9.4$, the transport time $(\sim p s)$ was much smaller than the fluorescence typical time of organic dyes $(\sim n s)$. We conclude hence that despite the strong scattering in the proposed samples, experiments using state-of-the-art techniques should be feasible.
[1] E. M. Purcell, Phys. Rev. 69, 37 (1946).

[2] W. Moerner and M. Orrit, Science 283, 1670 (1999); S. Weiss, ibid. 283, 1676 (1999).

[3] X. S. Xie and R. C. Dunn, Science 265, 361 (1994).

[4] R. A. L. Vallée, N. Tomczak, L. Kuipers, G. J. Vancso, and N. F. van Hulst, Phys. Rev. Lett. 91, 038301 (2003); R. A. L. Vallée, M. Van Der Auweraer, F. C. De Schryver, D. Beljonne, and M. Orrit, ChemPhysChem 6, 81 (2005).

[5] R. A. L. Vallée, M. Van der Auweraer, W. Paul, and K. Binder, Phys. Rev. Lett. 97, 217801 (2006).
[6] M. Sauer, J. Hofkens, and J. Enderlein, Handbook of Fluorescence Spectroscopy and Imaging: From Ensemble to Single Molecules (Wiley, New York, 2010).

[7] R. R. Chance, A. Prock, and R. Silbey, Adv. Chem. Phys. 37, 1 (1978).

[8] P. R. Berman, Cavity Quantum Electrodynamics (Academic, Boston, 1994).

[9] J. Martorell and N. M. Lawandy, Phys. Rev. Lett. 65, 1877 (1990); P. Lodahl, A. F. Van Driel, I. S. Nikolaev, A. Irman, K. Overgaag, D. Vanmaekelbergh, and W. L. Vos, Nature (London) 430, 654 (2004). 
[10] P. Anger, P. Bharadwaj, and L. Novotny, Phys. Rev. Lett. 96, 113002 (2006); S. Kühn, U. Håkanson, L. Rogobete, and V. Sandoghdar, ibid. 97, 017402 (2006); M. Ringler, A. Schwemer, M. Wunderlich, A. Nichtl, K. Kürzinger, T. A. Klar, and J. Feldmann, ibid. 100, 203002 (2008); A. G. Curto, G. Volpe, T. H. Taminiau, M. P. Kreuzer, R. Quidant, and N. F. van Hulst, Science 329, 930 (2010).

[11] B. O'regan and M. Grätzel, Nature (London) 353, 737 (1991).

[12] O. Painter, R. Lee, A. Scherer, A. Yariv, J. O'brien, P. Dapkus, and I. Kim, Science 284, 1819 (1999).

[13] S. Gottardo, R. Sapienza, P. D. Garcia, A. Blanco, D. S. Wiersma, and C. López, Nat. Photon. 2, 429 (2008).

[14] P. Michler, A. Kiraz, C. Becher, W. Schoenfeld, P. Petroff, L. Zhang, E. Hu, and A. Imamoglu, Science 290, 2282 (2000).

[15] B. A. van Tiggelen and S. E. Skipetrov, Phys. Rev. E 73, 045601 (2006).

[16] L. S. Froufe-Pérez, R. Carminati, and J. J. Sáenz, Phys. Rev. A 76, 013835 (2007); L. S. Froufe-Pérez and R. Carminati, Phys. Status Solidi A 205, 1258 (2008).

[17] A. Cazé, R. Pierrat, and R. Carminati, Phys. Rev. A 82, 043823 (2010).

[18] L. Sapienza, H. Thyrrestrup, S. Stobbe, P. Garcia, S. Smolka, and P. Lodahl, Science 327, 1352 (2010); S. Smolka, H. Thyrrestrup, L. Sapienza, T. B. Lehmann, K. R. Rix, L. S. Froufe-Pérez, P. D. García, and P. Lodahl, New J. Phys. 13, 063044 (2011).

[19] P. Ruijgrok, R. Wüest, A. Rebane, A. Renn, and V. Sandoghdar, Opt. Express 18, 6360 (2010).

[20] M. D. Birowosuto, S. E. Skipetrov, W. L. Vos, and A. P. Mosk, Phys. Rev. Lett. 105, 013904 (2010).

[21] R. Sapienza, P. Bondareff, R. Pierrat, B. Habert, R. Carminati, and N. F. van Hulst, Phys. Rev. Lett. 106, 163902 (2011).

[22] K. Joulain, R. Carminati, J.-P. Mulet, and J.-J. Greffet, Phys. Rev. B 68, 245405 (2003); R. Carminati, A. Caze, D. Cao, F. Peragut, V. Krachmalnicoff, R. Pierrat, and Y. De Wilde, Surf. Sci. Rep. 70, 1 (2015).

[23] R. Carminati, G. Cwilich, L. S. Froufe-Pérez, and J. J. Sáenz, Phys. Rev. A 91, 023807 (2015).

[24] B. Shapiro, Phys. Rev. Lett. 83, 4733 (1999).

[25] S. E. Skipetrov and R. Maynard, Phys. Rev. B 62, 886 (2000).

[26] R. Carminati and J. J. Sáenz, Phys. Rev. Lett. 102, 093902 (2009).

[27] M. Donaire, Phys. Rev. A 83, 022502 (2011).

[28] R. Ruppin, J. Chem. Phys. 76, 1681 (1982); H. Chew, ibid. 87, 1355 (1987); R. Carminati, J.-J. Greffet, C. Henkel, and J. Vigoureux, Opt. Commun. 261, 368 (2006); B. Rolly, B. Bebey, S. Bidault, B. Stout, and N. Bonod, Phys. Rev. B 85,
245432 (2012); M. Schmidt, R. Esteban, J. J. Sáenz, I. SuárezLacalle, S. Mackowski, and J. Aizpurua, Opt. Express 20, 13636 (2012).

[29] G. Scalia and F. Scheffold, Opt. Express 23, 29342 (2015).

[30] N. de Sousa, J. J. Sáenz, A. García-Martín, L. S. Froufe-Pérez, and M. I. Marqués, Phys. Rev. A 89, 063830 (2014).

[31] S. E. Skipetrov and I. M. Sokolov, Phys. Rev. Lett. 112, 023905 (2014).

[32] R. Rezvani Naraghi, S. Sukhov, J. J. Sáenz, and A. Dogariu, Phys. Rev. Lett. 115, 203903 (2015).

[33] C. L. Briant and J. J. Burton, J. Chem. Phys. 63, 2045 (1975); R. S. Berry, J. Jellinek, and G. Natanson, Phys. Rev. A 30, 919 (1984); J. Honeycutt and H. Andersen, J. Phys. Chem. 91, 4950 (1987); P. Labastie and R. L. Whetten, Phys. Rev. Lett. 65, 1567 (1990); D. J. Wales and R. S. Berry, ibid. 73, 2875 (1994).

[34] N. de Sousa, J. J. Sáenz, F. Scheffold, A. García-Martín, and L. S. Froufe-Pérez, J. Phys. Condens. Matter 28, 135101 (2016).

[35] D. V. van Coevorden, R. Sprik, A. Tip, and A. Lagendijk, Phys. Rev. Lett. 77, 2412 (1996).

[36] E. Yablonovitch, Phys. Rev. Lett. 58, 2059 (1987).

[37] R. Sprik, B. A. Van Tiggelen, and A. Lagendijk, Europhys. Lett. 35, 265 (1996); P. de Vries, D. V. van Coevorden, and A. Lagendijk, Rev. Mod. Phys. 70, 447 (1998).

[38] D. P. Landau and K. Binder, A Guide to Monte Carlo Simulations in Statistical Physics (Cambridge University Press, Cambridge, 2014).

[39] N. Ashcroft and J. Lekner, Phys. Rev. 145, 83 (1966).

[40] J. Cox, R. Farrell, R. Hart, and M. Langham, J. Physiol. 210, 601 (1970).

[41] L. F. Rojas-Ochoa, J. M. Mendez-Alcaraz, J. J. Sáenz, P. Schurtenberger, and F. Scheffold, Phys. Rev. Lett. 93, 073903 (2004); F. Scheffold and R. Cerbino, Curr. Opin. Colloid Interface Sci. 12, 50 (2007).

[42] M. Reufer, L. F. Rojas-Ochoa, S. Eiden, J. J. Sáenz, and F. Scheffold, Appl. Phys. Lett. 91, 171904 (2007); P. D. García, R. Sapienza, Á. Blanco, and C. López, Adv. Mater. 19, 2597 (2007); N. Muller, J. Haberko, C. Marichy, and F. Scheffold, Adv. Opt. Mat. 2, 115 (2014).

[43] S. Liew et al., Opt. Express 19, 8208 (2011); L. Cortese, L. Pattelli, F. Utel, S. Vignolini, M. Burresi, and D. S. Wiersma, Adv. Opt. Mater. 3, 1337 (2015).

[44] L. Novotny and B. Hecht, Principles of Nano-optics (Cambridge University Press, Cambridge, 2012).

[45] E. M. Purcell and C. R. Pennypacker, Astrophys. J. 186, 705 (1973).

[46] N. de Sousa, L. S. Froufe-Pérez, J. J. Sáenz, and A. GarcíaMartín, Sci. Rep. 6, 30803 (2016). 